\title{
On representations of the maximal unramified Galois extension of a field of positive characteristic
}

\author{
Hidenori KATSURADA \\ (Received February 3, 1986, Revised February 19, 1987)
}

\section{Introduction}

The aim of this paper is to give an expression of representations of the Galois group for the maximal unramified extension of a field of positive characteristic. In fact, we express the set of representations in a group theoretic means.

To be more precise, let $K$ be a field containing an algebraically closed field $k$ of positive characteristic $p$. Let $T$ be the set of all equivalence classes of discrete valuations of $K$ trivial on $k$ whose residue fields are isomorphic to $k$. For each $v$ of $T$, let $K_{v}$ be the completion of $K$ at $v$ and $O_{v}$ the integer ring in $K_{v}$. Put

and

$$
A=\prod_{v \in T} K_{v}, G_{n}(A)=\prod_{v \in T} G L_{n}\left(K_{v}\right),
$$

$$
O=\prod_{v \in T} O_{v}, \quad C_{n}=\prod_{v \in T} G L_{n}\left(O_{v}\right),
$$

where $G L_{n}$ denotes the general linear group of degree $n$. Let $K_{T}$ be the maximal Galois extension of $K$ which is unramified at every element of $T$. Then there is an imbedding $i_{v}$ of $K_{T}$ into $K_{v}$ because the residue field of $O_{v}$ is algebraically closed. Thus $K_{T}$ and $K$ can be imbedded into $A$ diagonally ; hence, $G L_{n}\left(K_{T}\right)$ and $G L_{n}(K)$ can be regarded as subgroups of $G_{n}(A)$. We use the same symbol $i_{v}$ to denote the imbedding of $G L_{n}\left(K_{T}\right)$ into $G L_{n}\left(K_{v}\right)$ induced by $i_{v}: K_{T} \rightarrow K_{v}$. Put

$$
G_{n}=C_{n} \backslash G_{n}(A) / G L_{n}(K) .
$$

For each element $u$ of $G_{n}(A)$, we denote by $[[u]]$ the double coset $C_{n} u G L_{n}(K)$. For each commutative ring $R$ of characteristic $p$, and for each p-power $q$, we can define an endomorphism $f_{q}$ of $M_{n}(R)$ by $\left(a_{i j}\right) \longmapsto\left(a_{i j}^{q}\right)$. This endomorphism induces a map from $G_{n}$ into itself, which will be again denoted by $f_{q}$. We often write $f=f_{q}$ if no confusion arises. We denote by 
$G_{n}^{f}$ the set of all $f$-fixed points of $G_{n}$. For each group $G$, let $\operatorname{Rep}\left(G, G L_{n}\right.$ $\left(F_{q}\right)$ ) be the set of all $G L_{n}(k)$-equivalence classes of representations of $G$ into $G L_{n}(k)$ whose images are isomorphic to some subgroups of $G L_{n}\left(F_{q}\right)$. Then our main result in this paper is

THEOREM 1. Assume that the intersection $O \cap K$ in $K$ coincides with $k$. Then there exists a bijection $\phi$ from $\operatorname{Rep}\left(G a l\left(K_{T} / K\right), G L_{n}\left(F_{q}\right)\right)$ onto $G_{n}^{f}$.

We shall prove this theorem in $\S 2$. When $K$ is a function field, we can express $\operatorname{Rep}\left(\operatorname{Gal}\left(K_{T} / K\right), G L_{n}\left(F_{q}\right)\right)$ in terms of adeles using Theorem 1. We shall explain this in more details. Let $X$ be an algebraic variety over $k$. Assume that $X$ is complete and normal. We take as $K$ the function field of $X$ over $k$. Then the set $T$ can be regarded as the set of all equivalence classes of valuations of prime divisors on $X$. Let $G L_{n}(K)_{A}$ be the adelization of $G L_{n}(K)$, and put

$$
G_{n}^{\prime}=C_{n} \backslash G L_{n}(K)_{A} / G L_{n}(K) .
$$

Then the assumptions of Theorem 1 are verified, and we have

TheOREM 2. The bijection $\phi$ in Theorem 1 induces a bijection from Rep $\left(G a l\left(K_{T} / K\right), G L_{n}\left(F_{q}\right)\right)$ onto the set $G_{n}^{\prime}$ of all f-fixed points of $G_{n}^{\prime}$.

Now let $V_{n}$ be the set of all isomorphism classes of locally free sheaves on $X$ of rank $n$. Then the $q$-th power absolute Frobenius map on $X$ induces a map $f$ from $V_{n}$ into itself. Let $\pi_{1}(X)$ be the algebraic fundamental group of $X$. Then, as an equivalent statement to Theorem 2, we have

Corollary to Theorem 2.") There exists a bijection from $\operatorname{Rep}\left(\pi_{1}(X)\right.$, $G L_{n}\left(F_{q}\right)$ ) onto the set $V_{n}^{f}$ of all f-fixed points of $V_{n}$.

We shall prove these results in $\S 3$. We remark that this corollary is a slight generalization of a result of $\mathrm{H}$. Lange and U. Stuhler [5], § 1 .

\section{$\S 2$. Proof of Theorem 1}

Let $k$ be a field containing a finite field $F_{q}$ and $K$ a field containing $k$. For a moment, we do not assume $k$ to be algebraically closed as we have done in $\S 1$. We use the same notations as in the introduction if otherwise mentioned. We denote by $\bar{K}$ the algebraic closure of $K$, and let $K_{s}$ be the separable algebraic closure of $K$. For a subfield $L$ of $\bar{K}$, put

$$
G L_{n}(L)^{\prime}=\left\{a \in G L_{n}(L) ; a^{-1} f(a) \in G L_{n}(K)\right\} .
$$

*) This was also announced in Katsurada [3] for the case where $K$ is an algebraic function field of one variable over $k$. 
For each element $a=\left(a_{i j}\right)_{1 \leqq i, j \leqq n}$ of $G L_{n}(\bar{K})$, let $K(a)$ be the field generated over $K$ by all components of $a$. Then for each $a \in G L_{n}(\bar{K})^{\prime}$, we have $K(a)^{q}$ $K=K(a)$, where $K(a)^{q}$ is the image of $K(a)$ under the $q$-th power homomorphism. Thus $K(a)$ is a separable extension of $K$ (cf. Proposition 1 of Lang [4]). Since $a^{-1} f(a)$ belongs to $G L_{n}(K)$, we have

$$
\sigma(a)^{-1} f(\sigma(a))=a^{-1} f(a),
$$

where $\sigma(a)$ denotes the matrix $\left(\sigma\left(a_{i j}\right)\right)_{1 \leqq i, j \leqq n}$ for each element $\sigma$ of the Galois group $\operatorname{Gal}\left(K_{s} / K\right)$. Hence we have

$$
a \sigma(a)^{-1}=f\left(a \sigma(a)^{-1}\right) .
$$

This shows that $a \sigma(a)^{-1}$ belongs to $G L_{n}\left(F_{q}\right)$ for each $\sigma \in \operatorname{Gal}\left(K_{s} / K\right)$. Hence we can define a map $\rho_{a}$ from $\operatorname{Gal}\left(K_{s} / K\right)$ into $G L_{n}\left(F_{q}\right)$ by

$$
\sigma \longmapsto a \sigma(a)^{-1} \text {. }
$$

We remark that $K(a)$ is a Galois extension of $K$, and for each $\sigma \in$ Gal $\left(K_{s} / K\right)$,

$$
\rho_{a}(\sigma)=a \bar{\sigma}(a)^{-1},
$$

where $\bar{\sigma}$ denotes the image of $\sigma$ under the natural surjection $t: \operatorname{Gal}\left(K_{s} / K\right)$ $\longrightarrow \operatorname{Gal}(K(a) / K)$. Now for all $\sigma, \tau$ of $\operatorname{Gal}\left(K_{s} / K\right)$, we have

$$
\begin{aligned}
\rho_{a}(\sigma \tau) & =a(\sigma \tau(a))^{-1}=a(\sigma(\tau(a)))^{-1}=a\left(\sigma\left(\rho_{a}(\tau)^{-1} a\right)\right)^{-1} \\
& =a\left(\rho_{a}(\tau)^{-1} \sigma(a)\right)^{-1}=a \sigma(a)^{-1} \rho_{a}(\tau)=\rho_{a}(\sigma) \rho_{a}(\tau) .
\end{aligned}
$$

This implies that $\rho_{a}$ is a homomorphism from $\operatorname{Gal}\left(K_{s} / K\right)$ into $G L_{n}\left(F_{q}\right)$.

Now for a subfield $L$ of $\bar{K}$ containing $K$, put

$$
\overline{G L_{n}(L)}=G L_{n}(k) \backslash G L_{n}(L) / G L_{n}(K),
$$

and

$$
\overline{G L_{n}(L)^{\prime}}=\left\{G L_{n}(k) a G L_{n}(K) ; a \in G L_{n}(L)^{\prime}\right\} .
$$

For each element $a$ of $G L_{n}(L)$, we denote by [a] the double coset $G L_{n}(k)$ $a G L_{n}(K)$. Moreover for each representation $\rho$ of a group in $G L_{n}(k)$, we denote by $[\rho]$ the $G L_{n}(k)$-equivalence class of $\rho$. We note that two elements $a, b$ of $G L_{n}(\bar{K})^{\prime}$ belong to the same double coset if and only if $\rho_{a}$ and $\rho_{b}$ are $G L_{n}(k)$-equivalent. In fact, if $a$ and $b$ belong to the same double coset, we have

$$
b=\text { uav }
$$

with $u \in G L_{n}(k), v \in G L_{n}(K)$. Then, for each element $\sigma$ of $\operatorname{Gal}\left(K_{s} / K\right)$, 
we have

$$
\begin{aligned}
\rho_{b}(\sigma) & =b \sigma(b)^{-1}=u a v \sigma(u a v)^{-1}=u a \sigma(a)^{-1} u^{-1} \\
& =u \rho_{a}(\sigma) u^{-1} .
\end{aligned}
$$

Thus $\rho_{a}$ and $\rho_{b}$ are $G L_{n}(k)$-equivalent. Conversely, if $\rho_{a}$ and $\rho_{b}$ are $G L_{n}(k)$ -equivalent, there is an element $u$ of $G L_{n}(k)$ such that

$$
\rho_{b}(\sigma)=u \rho_{a}(\sigma) u^{-1}
$$

for all $\sigma \in \operatorname{Gal}\left(K_{s} / K\right)$. Put $v=a^{-1} u^{-1} b$. Then for each $\sigma$ of $\operatorname{Gal}\left(K_{s} / K\right)$, we have

$$
\begin{aligned}
& \sigma(v)=\sigma\left(a^{-1} u^{-1} b\right)=\sigma(a)^{-1} u^{-1} \sigma(b)=a^{-1} \rho_{a}(\sigma) u^{-1} \rho_{b}(\sigma)^{-1} b \\
& \quad=a^{-1} u^{-1} b=v .
\end{aligned}
$$

Hence, $v$ belongs to $G L_{n}(K)$. This implies that $a$ and $b$ belong to the same double coset. Thus we can define an injective map $\psi$ from $\overline{G L_{n}(\bar{K})^{\prime}}$ into $R e p$ $\left(\operatorname{Gal}\left(K_{s} / K\right), G L_{n}\left(F_{q}\right)\right)$ by

$$
[a] \longmapsto\left[\rho_{a}\right] \text {. }
$$

The following proposition is essentially obtained by Inaba [2], but the formulation and the proof are slightly different.

Proposition 3. $\psi$ is bijective.

ProOF. It suffices to show that $\psi$ is surjective. Let $[\rho]$ be an element of $\operatorname{Rep}\left(\operatorname{Gal}\left(K_{s} / K\right), G L_{n}\left(F_{q}\right)\right)$ and $L_{\rho}$ the field corresponding to the kernel of $\rho$. Then $\rho$ is factored through $\operatorname{Gal}\left(L_{\rho} / K\right)$ like

$$
\operatorname{Gal}\left(K_{s} / K\right) \stackrel{t}{\longrightarrow} \operatorname{Gal}\left(L_{\rho} / K\right) \stackrel{\bar{\rho}}{\longrightarrow} G L_{n}\left(F_{q}\right) .
$$

Since we have $F_{q} \subset K, \bar{\rho}$ gives an element of the set of 1-cocycles $Z^{1}$ $\left(\operatorname{Gal}\left(L_{\rho} / K\right), G L_{n}\left(L_{\rho}\right)\right)$. Since we have $H^{1}\left(\operatorname{Gal}\left(L_{\rho} / K\right), G L_{n}\left(L_{\rho}\right)\right)=\{1\}$ (for example, see Serre [6], Chp. X, §1), there is an element $a$ of $G L_{n}\left(L_{\rho}\right)$ such that $\bar{\rho}(\sigma)=a \sigma(a)^{-1}$ for all $\sigma \in \operatorname{Gal}\left(L_{\rho} / K\right)$. Put $\alpha=a^{-1} f(a)$. Then we have $\sigma(\alpha)=\alpha$ for all $\sigma \in \operatorname{Gal}\left(L_{\rho} / K\right)$, that is, $\alpha$ belongs to $G L_{n}(K)$. Moreover we have $\psi([a])=[\rho]$. This proves the surjectivity.

Now let $k, K$ be as in $\S 1$. Let $\phi^{\prime}$ be the inverse map of $\psi$. Let $\delta: G a l$ $\left(K_{s} / K\right) \longrightarrow \operatorname{Gal}\left(K_{T} / K\right)$ be the natural surjection. Then for each element [ $\rho$ ] of $\operatorname{Rep}\left(\operatorname{Gal}\left(K_{T} / K\right), G L_{n}\left(F_{q}\right)\right)$, we can take an element $a$ of $G L_{n}\left(K_{s}\right)^{\prime}$ such that $[a]=\phi^{\prime}\left(\left[\rho^{\circ} \delta\right]\right)$. (Recall $\left.G L_{n}(\bar{K})^{\prime}=G L_{n}\left(K_{s}\right)\right)$. Let $\rho_{a}$ be the homomorphism from $\operatorname{Gal}\left(K_{s} / K\right)$ into $G L_{n}\left(F_{q}\right)$ defined above. Then by our 
construction, we have $\psi([a])=\left[\rho_{a}\right]$. Thus two representations $\rho \circ \delta$ and $\rho_{a}$ are $G L_{n}(k)$-equivalent each other. Consequently, $a$ belongs to $G L_{n}\left(K_{T}\right)$. Thus $a$ belongs to $G_{n}(A)$, and we have $[[f(a)]]=[[a]]$ in $G_{n}$. Since $k$ is contained in $O_{v}$ for each $v \in T$, we can define a map $\phi$ from $R e p$ $\left(G a l\left(K_{T} / K\right), G L_{n}\left(F_{q}\right)\right)$ into $G_{n}^{f}$ by

$$
[\rho] \longmapsto[[a]] \text {. }
$$

Then to prove Theorem 1, it suffices to prove

THEOREM 1'. $\phi$ is bijective.

To prove this, we need the following fact.

Lemma 4. The map $\xi$ from $C_{n}$ into itself defined by $u \longmapsto f(u) u^{-1}$ is surjective.

Proof. Since we have $C_{n}=\prod_{v \in T} G L_{n}\left(O_{v}\right)$, it suffices to show that for each $v \in T$, the map

$$
G L_{n}\left(O_{v}\right) \ni u \longmapsto f(u) u^{-1} \in G L_{n}\left(O_{v}\right)
$$

is surjective. Let $M$ be the maximal ideal of $O_{v}$. Let $b$ be an element of $G L_{n}\left(O_{v}\right)$. We claim that we can take a sequence $\left\{a_{i}\right\}(i=0,1, \ldots)$ of $M_{n}\left(O_{v}\right)$ such that

$$
\begin{aligned}
& a_{0} \in G L_{n}\left(O_{v}\right), \\
& f\left(a_{i}\right) \equiv b a_{i} \bmod M^{i+1},
\end{aligned}
$$

and

$$
a_{i+1} \equiv a_{i} \bmod M^{i+1}
$$

In fact, since $O_{v} / M$ is algebraically closed, there is an element $a_{0}$ of $G L_{n}\left(O_{v}\right)$ such that

$$
b \equiv f\left(a_{0}\right) a_{0}^{-1} \bmod M
$$

(cf. Lang [4]). Assume that $a_{0}, \ldots, a_{i}$ are elements of $M_{n}\left(O_{v}\right)$ such that

and

$$
f\left(a_{j}\right) \equiv b a_{j} \bmod M^{j+1},
$$

$$
a_{j} \equiv a_{j-1} \bmod M^{j}
$$

for $1 \leqq j \leqq i$. Put $a_{i+1}=b^{-1} f\left(a_{i}\right)$. Then we have

$$
\begin{aligned}
& f\left(a_{i+1}\right)-b a_{i+1}=f\left(b^{-1}\right) f\left(f\left(a_{i}\right)\right)-b b^{-1} f\left(a_{i}\right) \\
& \equiv f\left(b^{-1}\right) f\left(b a_{i}\right)-f\left(a_{i}\right) \equiv 0 \bmod M^{i+2},
\end{aligned}
$$


and

$$
a_{i+1}-a_{i}=b^{-1}\left(f\left(a_{i}\right)-b a_{i}\right) \equiv 0 \bmod M^{i+1} .
$$

Thus by induction, we obtain a sequence $\left\{a_{i}\right\}$ with the required properties.

Since $O_{v}$ is a complete ring, this sequence $\left\{a_{i}\right\}$ has a limit a in $M_{n}\left(O_{v}\right)$. It follows from the construction that we have

$$
a \equiv a_{0} \bmod M \text {, }
$$

and

$$
f(a) \equiv b a \bmod M^{i}
$$

for $i=1,2, \ldots$ Thus a belongs to $G L_{n}\left(O_{v}\right)$, and we have $b=f(a) a^{-1}$. This proves the lemma.

Proof of Theorem 1'. First we prove the injectivity of $\phi$. Let $\left[\rho_{i}\right]$ $(i=1,2)$ be an element of $\operatorname{Rep}\left(\operatorname{Gal}\left(K_{T} / K\right), G L_{n}\left(F_{q}\right)\right)$, and let $a_{i}$ be an element of $G L_{n}\left(K_{T}\right)^{\prime}$ such that $\left[\left[a_{i}\right]\right]=\phi\left(\left[\rho_{i}\right]\right)$. Assume that we have

$$
\left[\left[a_{1}\right]\right]=\left[\left[a_{2}\right]\right] \text {. }
$$

Then we have

(1) $a_{i}^{-1} f\left(a_{i}\right) \in K$,

for $i=1,2$, and we have

(2) $a_{2}=a a_{1} b$

with $a \in C_{n}$, and $b \in G L_{n}(K)$. Then by (1) and (2), there is an element $\beta$ of $G L_{n}(K)$ such that

(3) $a^{-1} f(a)=a_{1} \beta a_{1}^{-1}$.

Now, as remarked at the beginning of this section, for each $\sigma \in G a l$ $(K(a) / K)$, there is an element $\tau_{\sigma}$ of $G L_{n}\left(F_{q}\right)$ such that

(4) $\sigma\left(a_{1}\right)=\tau_{\sigma} a_{1}$.

Thus by (3) and (4), for each element $\sigma$ of $\operatorname{Gal}\left(K\left(a_{1}\right) / K\right)$, we have

(5) $\sigma\left(a_{1} \beta a_{1}^{-1}\right) \in C_{n}$.

Put $a_{1} \beta a_{1}^{-1}=\left(u_{i j}\right)_{1 \leqq i, j \leqq n}$. Let $f_{i j}(X)$ be the irreducible polynomial of $u_{i j}$ over $K$. Then by (5), $f_{i j}(X)$ belongs to $O_{v}[X]$ for all $v \in T$. Since we have $O \cap K=k, f_{i j}(X)$ belongs to $k[X]$. Since $k$ is algebraically closed, $u_{i j}$ belongs to $k$. This implies that $a^{-1} f(a)$ belongs to $G L_{n}(k)$. Thus a belongs to $G L_{n}(k)$ by Corollary to Theorem 1] of [4]. Hence by Proposition 3 , we have $\left[\rho_{1}\right]=\left[\rho_{2}\right]$. This shows that $\phi$ is injective.

Now to prove the surjectivity of $\phi$, let $[[a]]$ be an f-fixed point of $G_{n}$. Then we have

$$
f(a)=u a d
$$

with $u \in C_{n}, d \in G L_{n}(K)$. By Lemma 4, there is an element $w$ of $C_{n}$ such 
that

$$
f(w) w^{-1}=u .
$$

Then $b=w^{-1} a$ satisfies

$$
b^{-1} f(b)=d .
$$

Now let $c$ be an element of $G L_{n}(\bar{K})$ such that

$$
c^{-1} f(c)=d .
$$

Let $T(L)$ be the set of all extensions of elements of $T$ to the field $L=K(c)$. For each element $v$ of $T(L)$, let $j_{v}$ be the imbedding of $G L_{n}(L)$ into $G L_{n}\left(K_{v}\right)$. Then $j_{v}(c) b^{-1}$ belongs to $G L_{n}\left(F_{q}\right)$. Thus $j_{v}(c)$ belongs to $G L_{n}\left(K_{v^{\prime}}\right)$, where $v^{\prime}$ is the restriction of $v$ to $K$. This implies that $K(c)$ is contained in $K_{T}$, and we have

$$
[[c]]=[[a]]
$$

in $G_{n}$. Put $[\rho]=\psi([c])$. Then there is a homomorphism $\bar{\rho}$ from $G a l$ $\left(K_{T} / K\right)$ into $G L_{n}\left(F_{q}\right)$ such that $\rho=\bar{\rho}^{\circ} \delta$. Since we have $[c]=\phi^{\prime}\left(\left[\bar{\rho}^{\circ} \delta\right]\right)$, we have $[[a]]=[[c]]=\phi([\bar{\rho}])$ by our construction. This proves the surjectivity of $\phi$.

\section{$\S 3$. Proof of Theorem 2 and its corollary}

Now let $K$ be the function field of a complete normal irreducible algebraic variety $X$ over $k$. To prove Theorem 2, it suffices to show the following fact.

Proposition 5. Let $G_{n}^{f}$ and $G_{n}^{\prime f}$ be as in $\S 1$. Then we have $G_{n}^{f}=G_{n}^{\prime}$.

Proof. Clearly we have $G_{n}^{\prime f} \subset G_{n}^{f}$. We prove the converse inclusion. Let $[[a]]$ be an element of $G_{n}^{f}$. Then we have $f(a)=$ uad with $u \in C_{n}, d \in$ $G L_{n}(K)$. Let $c$ an element of $G L_{n}(\bar{K})$ such that $c^{-1} f(c)=d$. Then $c$ belongs to $G L_{n}\left(K_{T}\right)$, and we have $[[c]]=[[a]]$ (see the proof of Theorem $\left.1^{\prime}\right)$. Since $K(c)$ is a finite extension of the function field $K$ of a normal variety, we have $i_{v}(c) \in G L_{n}\left(O_{v}\right)$ for all except finitely many elements $v$ of $T$. This shows that $c$ belongs to $G L_{n}(K)_{A}$. Hence [[a]] belongs to $G_{n}^{\prime}$. This proves the assertion.

Proof of Corollary to Theorem 2. First we show that there exists a bijection from $G_{n}^{\prime f}$ onto $V_{n}^{f}$. We use the same symbol $P$ to denote the equivalence class of the valuation of a prime divisor $P$ on $X$. Fix a vector space $V$ over $k$ together with a basis $e_{1}, \ldots, e_{n}$. Let $M_{O}=\left\{M_{O, P}\right\}_{P}$ be the 
family of $O_{P}$-lattices in $\underset{K}{\otimes} K_{P}$ such that

$$
M_{O, P}=O_{P} e_{1} \oplus \ldots \oplus O_{P} e_{n}
$$

for all $P \in T$. We use the same symbol $i_{P}$ to denote the homomorphism from $V$ into $V \underset{K}{\otimes} K_{P}$ induced by the imbedding $i_{P}: K_{T} \longrightarrow K_{P}$. Then for each $a=$ $\left(a_{P}\right)$ of $G L_{n}(K)_{A}$, we define a presheaf $E(a)$ on $X$ by $\Gamma(U, E(a))=\{x \in V$; $i_{P}(x) \in a_{P}^{-1} M_{O, P}$ for all $\left.P \subset U\right\}$, where $U$ is an open subset of $X$. It is easily checked that $E(a)$ is a locally free sheaf of rank $n$. Moreover we have $E$ $(a) \cong E(b)$ if we have $[[a]]=[[b]]$ in $G_{n}^{\prime}$. Thus we can define a map $\theta$ from $G_{n}^{\prime}$ into $V_{n}$ by

$$
[[a]] \longmapsto \text { the isomorphism class of } E(a) \text {. }
$$

It is easily checked that $\theta$ is a bijection (cf. $\S 1$ of G. Harder and D. A. Kazhdan [1] $)$. Now by our construction, we have $\theta\left(G_{n}^{\prime f}\right)=V_{n}^{f}$. On the other hand, we have $\operatorname{Gal}\left(K_{T} / K\right) \cong \pi_{1}(X)$. Thus the assertion follows immediately from Theorem 2 .

AcKNowledgements. The author would like to thank the referee for his valuable advice.

\section{References}

[1] G. HARder and D. A. KAZHDAN, Automorphic forms $G L_{2}$ over function fields (after V. G. Drinfeld), Proceedings of Symposia in Pure Math. 33, part 2, AMS (1979), 357-379.

[ 2 ] E. InABA, On generalized Artin Shreier equations, Nat. Sci. Rep. Ochanomizu Univ. 13 (1962), 1-13.

[ 3 ] H. Katsurada, On unramified $S L_{2}\left(F_{4}\right)$-extensions of an algebraic function field, Proc. Japan Acad. 56 (1980), 36-39.

[ 4 ] S. LANG, Algebraic groups over finite fields, Amer. J. of Math. 78 (1965), 553-563.

[ 5 ] H. LANGE and U. STUhLER, Vektorbündel auf Kurven und Darstellungen der algebraichen Fundamentalgruppe, Math. Z. 156 (1977), 73-83.

[6] J. P. SERRE, Corps locaux, Herman, Paris (1962). 